Ann. Biol. anim., Bioch., Biophys., I965, 5 (4), 445-450.

\title{
GROUPES SANGUINS ET SÉRIQUES DES ANES
}

\author{
Luba PODLIACHOUK, Marie KAMINSKI et Z. DABCZEWSKI \\ Institut Pasteur et Centre national de la Recherche scientifique, Paris \\ Agricultural Institute, Dublin
}

\section{SOMMAIRE}

Cette étude a porté sur 89 ânes appartenant à deux types différent par des caractères morphologiques: l'un de l'Est (Bog of Allen), le second (Connemara) de l'Ouest de l'Irlande.

Deux antigènes érythrocytaires asins, $\mathrm{B}$ et $\mathrm{N}$, ont été étudiés : pour l'antigène $\mathrm{B}$ les fréquences ne diffèrent pas sensiblement d'un type à l'autre - 0,68 et 0,62 ; tandis que pour l'antigène $\mathrm{X}$ la fréquence est plus élevée dans le type Connemara que dans Bog of Allen -.. 0,33 et o,23.

La présence d'anti-B a été décélée deux fois dans le sérum de 30 animaux $B$ négatifs et celle d'anti- 9 fois dans le sérum de 66 animaux $\mathrm{N}$ négatifs.

Pami les constituants sériques décélés en électrophorèse en gel d'amidon, des bandes situées dans les zones des $\alpha$-globulines rapides, des $\beta$-globulines rapides, des $\beta$-globulines lentes, ainsi qu'une estérase rapide présentent des variations quantitatives et qualitatives.

Six phénotypes des $\beta$-globulines rapides et trois des $\beta$-globulines lentes ont été décélés dans les sérums examinés. I,eurs fréquences sont différentes dans les deux types d'ânes.

\section{INTRODUC'TION}

Le présent travail consacré à l'espèce Ane (Equus Asinus) fait suite aux études sur les groupes sanguins des Équidés, effectuées par Podłiachouk, ainsi qu'aux travaux de Kaminski sur les protéines sériques.

Depuis l'observation de LANDSTEINER et VAN DER SchEER (I924), que le sérum des ânes agglutine les hématies du cheval, la première étude des antigènes érythrocytaires asins a été faite par PodLrachouk et Exquem (I953). L'examen de 78 ânes et de 135 mulets a permis d'individualiser un premier antigène érythrocytaire des ânes, nommé $B$. Les antigènes des Équidés, Cheval et Ane, pouvant être décelés chez leur hybride, le Mulet, ont été, au fur et à mesure de leur découverte, désignés par des lettres majuscules (sans séparer les antigènes des deux espèces).

La transmission héréditaire de l'antigène $B$ a été étudiée chez $I 6$ familles d'ânes comprenant 23 produits (PoDLIACHOUK, I957). Cet antigène est transmis suivant les lois de l'hérédité mendelienne. De même que la plupart des antigènes du Cheva1, il est déterminé par un couple d'allèles avec dominance de la présence sur l'absence. 
La fréquence de l'antigène $B$ varie avec la race étudiéa (PondIAchouk, I957): elle est la plus élevée dans la race poitevine et très faible dans la race catalane. L'étude des familles permet de supposer que les animaux de race catalane pure sont $B$ négatifs, ceux de race poitevine pure sont $B$ positifs homozygotes, ceux de race pyrénéenne pure $B$ positifs hétérozygotes. Chez 47 ânes de Tunisie et 26 de Paris, populations hétérogènes, la fréquence du facteur $B$ est respectivement de 0,74 et 0,65 . Par contre, 5 baudets de Niort de race poitevine (reproducteurs de mulets) sont tous B positifs. 3 I ânes provenant de la République du Tchad (Podirachouk et Quevar, I96I), représentant une population apparemment homogène, appartiennent tous à un type morphologique unique, répandu en Afrique Noire; leur étude sérologique a permis de déceler un deuxième antigène érythrocytaire asin, nommé M. Les fréquences des antigènes $\mathrm{B}$ et $\mathrm{M}$ chez ces animaux étaient respectivement $0,5^{8}$ et 0,89 . Parmi Ig ânes d'Alger (Podliachouk et Rioche, I962) la fréquence de $B$ est 0,68 , ce qui est voisin de valeurs trouvées pour d'autres populations hétérogènes. L'antigène $\mathrm{M}$ était présent chez tous les ânes d'Alger examinés.

Un troisième facteur asin, $\mathrm{N}$, a été découvert (PoDliachouk, I964), en immunisant des lapins à l'aide des hématies du mulet.

L'analyse immunochimique des protéines sériques de l'Ane (KAMINSKI, I964; Podliachouk et Kaminski, I964), effectliée à l'aide d'immunsérums de Lapin anti-sérum de Cheval, d'Ane, du Mulet et du Bardot, a permis de distinguer dans le diagramme immunoélectrophorétique au moins deux lignes de $\gamma$-globulines, quatre de $\beta$-globulines dont la transferrine, plusieurs $\alpha_{2}$ et $\alpha_{1}$ globulines, l'albumine et le $\delta\left({ }^{1}\right)$.

L'étude des estérases, faite en gel de gélose et gel d'amidon (Kamixski et Gajos, I964), a démontré dans le sérum de 60 ânes, conservé congelé pendant 3 ans au minimum, la présence de cholinestérase sérique correspondant à une $\alpha$-globuline rapide.

Le présent travail est une étude des groupes sanguins et des constituants sériques des ânes de l'Irlande.

Les ânes ont été amenés en Irlande par des Celtes il y a environ 2.500 ans. Il est probable qu'ils étaient apparentés aux ânes français et espagnols.

Actuellement on rencontre en Irlande deux types d'ânes séparés géographiquement : type Bog of Allen de la région de 1'Est et type Connemara de 1'Ouest de 1'Ile.

Au point de vue morphologique les ânes de Bog of Allen sont un peu plus grands que ceux de Connemara; ceci est probablement dî̀ aux meilleures conditions de vie. Ces deux populations ne sont pas croisées entre elles.

\section{MATÉRIEI, ETT TECHNIQUES}

Le sang a été prélevé chez les ânes de deux sexes :

65 animaux du type Bog of Allen, 24 animaux du type Connemara.

Les échantillons de sang ont été prélevés stérilement d'une part sur solution d'Alsever et d'autre part sans anticoagulant. Les suspensions globulaires et les sérums ont été expédiés par avion à Paris.

Les échantillons globulaires étaient conservés à $+4^{\circ} \mathrm{C}$ et les sérums congelés à $-20^{\circ} \mathrm{C}$.

(1) $\delta$ (lettre grecque rho) provient du mot rapide et désigne d'aprés Grabar et BurTin (ig6o) les protéines migrant plus rapidement que l'albumine dans le sérum humain soumis à l'analyse immuno-électrophorétique. 


\section{Détermination des groupes sanguins}

On a utilisé deux anticorps de référence, décelant les antigènes $\mathrm{B}$ et $\mathrm{N}$. L'antigène $\mathrm{M}$ n'a pu être recherché, l'anticorps correspondant étant épuisé. Les anti-B étaient soit des isoagglutinines naturelles, soit des hétéroagglutinines naturelles provenant du sérum de Mulet ou de Cheval. Les anti-N étaient des isoagglutinines naturelles ou des hétéro-immun-sérums de lapin anti-hématies de Mulet. Les sérums de référence étaient conservés congelés à $-20^{\circ} \mathrm{C}$ ou lyophilisés.

La réaction d'agglutination est effectuée en tubes à hémolyse. Les globules rouges sont lavés deux fois avec du ClNa à $0,9 \mathrm{p}$. Ioo et mis en suspension à I p. Ioo. Les sérums sont mis en présence de la suspension de globules rouges à raison de $0,05 \mathrm{ml}$ ( $\mathrm{c}$ goutte) de sérum pour $0,05 \mathrm{ml}$ ( $\mathrm{I}$ goutte) de globules rouges. La première lecture est faite, après 30 minutes de contact sans agitation à la température du laboratoire, à l'œil nu à l'aide d'un miroir concave. La deuxième lecture, faite après agitation et centrifugation, pendant une minute à I 800 tours/minute, permet en général de confirmer on d'infirmer les résultats difficiles à interpréter.

\section{Electrophorèse en gel d'amidon}

Dix échantillons de sérum sont soumis simultanément à une migration horizontale avec un système discontinu de tampons; pour le gel on utilise 5,5 volumes de tampon Triscitrate $(8,67 \mathrm{~g}$ de Tris + I, $33 \mathrm{~g}$ d'acide citrique, par litre) + I volume de tampon borate de Li ( $1 \mathrm{I}, 8 \mathrm{~g}$ d'acide borique par litre + hydroxyde de lithium environ $3 \mathrm{~g}$ pour amener le $\mathrm{pH}$ à 8,7 ). Dans les cuves on utilise le tampon borate de Li seul.

Le voltage est de $10 \mathrm{~V} / \mathrm{cm}$ sous $100 \mathrm{~mA}$ ce qui donne une migration de l'albumine de $10 \mathrm{~cm}$ en 6 heures. Les plaques sont refroidies par un dispositif à eau courante par en-dessous et à l'aide de vessies à glace par dessus. Le gel épais de $9 \mathrm{~mm}$ est coupé en trois tranches : l'une (celle du fond) colorée par le Noir Amide pour les protéines, les deux autres servant à localiser soit les lipides, soit les estérases, soit les transferrines, par les réactions spécifiques (KAMINSKI et GAJOS, 1964).

\section{RÉSULTATS}

Les fréquences des antigènes érythrocytaires $B$ et $N$, trouvées chez les 65 ânes du type de Bog of Allen sont respectivement de 0,68 et 0,23 , celles trouvées chez les 24 ânes du type Connemara 0,62 et 0,33 .

Dans le sérum de ces ânes on a décelé la présence d'isoagglutinines naturelles : l'anti-B 2 fois sur 30 animaux $B$ négatifs et l'anti-N 9 fois sur 66 animaux $N$ négatifs. Un de ces anti-N est très puissant : son titre est de I/I28. Il faut noter, en outre, dans 2 I sérums la présence d'isoagglutinines très faibles dont la spécificité n'a pas pu être déterminée; certaines de ces isoagglutinines sont probablement des anti-N.

Les protéinogrammes des sérums d'ânes montrent 8 à I6 bandes; plusieurs groupes de bandes apparaissent comme des systèmes variables soit qualitativement - présence ou absence, soit quantitativement — par l'intensité de coloration.

Dans la région des $\beta$-globulines rapides un groupe composé de 4 bandes constitue le système variable le plus important; ces bandes ont été désignées $3,4,5,6$ (KAMINSKI, I964).

Un groupe de bandes de migration plus lente, composé de 4 bandes, présentes toujours deux par deux, est désigné provisoirement $A, B, C, D$.

Le marquage du sérum d'âne au ${ }^{59} \mathrm{Fe}$ et l'analyse autoradiographique ont permis de constater que parmi les bandes des $\beta$-globulines rapides une ne fixe pas le fer, alors que les $\beta$-globulines lentes fixent le fer.

Des variations ont été observées parmi les $\alpha$-globulines rapides et les préalbumines. 
La figure I montre la disposition de bandes et les phénotypes de $\beta$-globulines rapides relevés dans $5 \mathrm{I}$ sérums de Bog of Allen et 24 sérums de Connemara. 14 échantillons de sérum du type Bog of Allen sont arrivés hémolysés, ce qui rendait impossible la lecture des bandes de $\beta$-globulines, recouvertes par une large tache d'hémoglobine. L'analyse des $\beta$-globulines lentes a été faite sur 59 sérums de $B o g$ of Allen et 24 de Connemara.

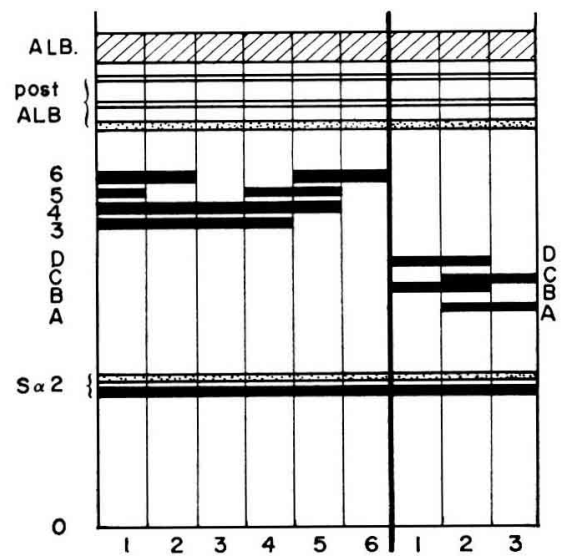

Fig. I - Electrophorégramme de sérum d'ânes:

6 phénotypes de $\beta$-globulines rapides et 3 phénotypes de $\beta$-globulines lentes.

Les fréquences de phénotypes des $\beta$-globulines rapides dans deux types d'ânes sont présentées au tableau I.

TABLEAU I

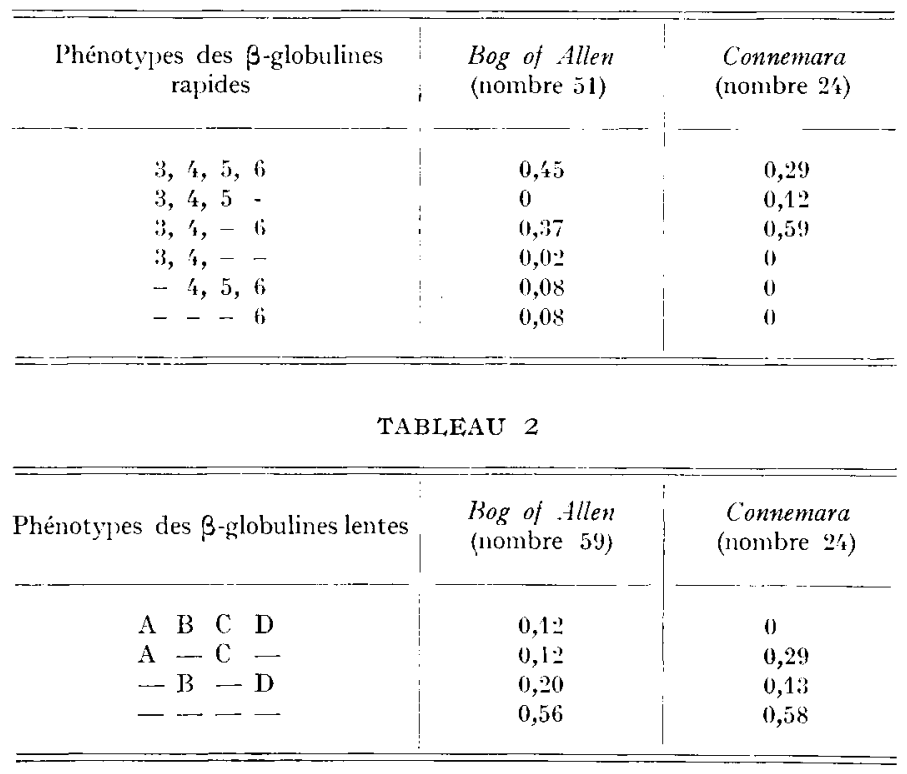

Les frẽquences de phénotypes des $\beta$-globulines lentes sont présentées au tableau 2 


\section{DISCUSSION E'T CONCLUSION}

Nous avons constaté que dans les deux types d'ânes irlandais les fréquences de 1'antigène $B$ sont très similaires $(0,68$ et 0,62$)$; elles ne diffèrent pas beaucoup des fréquences trouvées dans d'autres populations hétérogènes des ânes : Tunisie 0,74, Paris o,65 (Podliachouk, I957), 'Tchad 0,58 (Podliachouk et Queval, i96i) et Alger o,68 (Podliachouk et Rroche, r962). La fréquence de l'antigène $\mathrm{N}$ est plus élevée dans le type Connemara $(0,33)$ que dans le type Bog of Allen $(0,23)$.

Les isoagglutinines naturelles sont relativement rares; chez les animaux $B$ négatifs anti-B a été décelé 2 fois sur 30 ânes d'Irlande, $\mathrm{I}$ fois sur $2 \mathrm{I}$ de France et de Tunisie, 2 fois sur 6 ânes d'Alger; par contre chez les ânes du Tchad l'anti-B a été trouvé 7 fois sur I $3_{3}$ animaux $B$ négatifs.

La connaissance des groupes sanguins des ânes a permis de constater que l'jctère hémolytique du muleton est dû non seulement à l'immunisation de juments mulassières " tachées de jaunisse » vis-à-vis des antigènes spécifiques d'espèce Ane (CAROLI et BESSIS, I947) mais aussi vis-à-vis de l'antigène érythrocytaire asin B. Il est très probable que les autres antigènes érythrocytaires interviennent également.

En ce qui, concerne les constituants sériques il samble que les deux populations d'ânes présentent des différences dans les fréquences des phénotypes aussi bien des $\beta$-globulines rapides que des $\beta$-globulines lentes.

Dans une étude antérieure (KAMrnski et Gajos, I964), faite sur le sérum d'ânes conservé pendant plus de 3 ans, nous avons constaté pour 62 animaux étudiés l'absence régulière d'une estérase présente dans tous les sérums de chevaux, même très anciens. Au cours du présent travail ca constituant a été observé chez la plupart des animaux; la tâche est moins intensément coloré e que pour des sérums de chevaux et migre légèrement moins vite. Comme les sérums analysés étaient relativement frais et que le système de tampons a été modifié depuis le travail précédent, il ne nous est pas encore possible d'affirmer s'il s'agit d'une caractéristique particulière aux ânes irlandais ou bien d'une différence d'ordre technique.

\section{Reçu pour publication en juillet 1965}

\section{SUMMARY}

\section{BLOOD GROUPS AND SERUM COMPONENTS IN DONKEYS}

Sixty-five donkeys from the eastern part of Ireland (type Bog of Allen) and 24 from the western part (type Connemara) differing between them by some morphological characters, were studied.

Two erytrocyte antigens $\mathrm{B}$ et $\mathrm{N}$, were detected : the frequencies of $\mathrm{B}$ are similar in the two types $(0,68-0,62)$ while $\mathrm{N}$ is more frequent in the Connemara than in Bog of Allen $(0,33$ and 0,23$)$.

Anti-B antibodies were found in 2 sera out of $30 \mathrm{~B}$ negative animals; anti- $\mathrm{N}$ in 9 out of 66.

In starch gel electrophoresis serum protein polymorphism was observed for the fast-migrating $\alpha$-globulins, the fast and the slow $\beta$-globulins. The variations are both qualitative and quantitative.

Six phenotypes of fast $\beta$-globulins and 3 phenotypes of the slow $\beta$-globulins were observed. The frequencies differed for the two types of donkeys. 


\section{REMERCIEMENTS}

Nous remercions très vivement M. le Docteur J. M. Fine, Service d'Immunochimie et M. le Docteur P. Amouch, Service des Isotopes du Centre National de Transfusion Sanguine à Paris, pour le marquage des sérums d'ânes au fer radioactif et pour l'analyse autoradiographique.

\section{RÉFÉRENCES BIBLIOGRAPHIQUES}

Caroli J., Bessis M., i947. Recherches sur la cause de l'ictère grave familial des mule tons. Rev. Hémat. 2, 207-228.

Grabar P., Burtin P., ig60. Analyse immuno-électrophorétique, p. 93, Ed. Masson, Paris.

Kaminski M., I964. Serum proteins in Equidae : species, race and individual differences. IX Eur. Conf. Animal Blood Groups, Prague.

Kaminski M., Gajos E., rg64. Comparative examination of Carboxylic Esterases in Sera of Horse, Donkey and their Hybrids. Nature, G. B., 20I, 7 6 6-7I8.

LANDSteiner K., Van Der Scheer J., 1924. Serological examination of a species-hybrid. J. Immunol., 9, $2 \mathrm{I} 3^{-226 .}$

Podlachouk L., I957. Les antigènes de groupes sanguins des Équidés et leur transmission héréditaire. Thèse Doct. ès-Sci., Paris.

Podliachouk L., ig64. Blood groups of Equidae. IX Eur. Conf. Animal Blood Groups, Prague.

Podlachouk L., EYQuem A., 1953. Les groupes sanguins des Équidés. III. Les groupes sanguins des Anes. Ann. Inst. Pasteur, 84, 966-968.

Podliachouk L., Kaminski M., 1964. Études immunologiques sur les Équidés. Ann. Inst. Pasteur 106, 497-50 I.

PoDliachouk L., Queval R., i96г. Les groupes sanguins des Équidés du Tchad. Ann. Inst. Pasteur, 100, I $33^{-1} 3^{6 .}$

Podliachouk L., Rioche M., ig62. (Données non publiées). 